\title{
Does Wage Persistence Matter for Employment Fluctuations? Evidence from Displaced Workers
}

\author{
B Y JOHANNES F. SCHMIEDER AND TILL VON WACHTER \\ WEB APPENDIX
}

Table A-1 : Sample Means - SIPP

\begin{tabular}{lc}
\hline & $(1)$ \\
& SIPP \\
\hline Number of observations & 86703 \\
\hline Panel A: Outcome variables & \\
Layoff or fired & 0.060 \\
Quit & 0.067 \\
Job ends & 0.18 \\
Industry switch between first and last month & 0.22 \\
Occupation switch between first and last month & 0.24 \\
Hourly wage first month & 14.0 \\
& {$[11.9]$} \\
Hourly wage last month & 15.8 \\
& {$[19.7]$} \\
\hline Panel B: Demographics & \\
Tenure & 8.29 \\
& {$[8.27]$} \\
Age & 39.1 \\
& {$[11.0]$} \\
High School Grad & 0.33 \\
Some College & 0.28 \\
College Degree & 0.24 \\
Post-College & 0.047 \\
Married & 0.65 \\
Non-White & 0.23 \\
\hline Panel C: Unemployment Rates & \\
Unemployment at beginning of job & 6.27 \\
held in first month of panel & {$[1.88]$} \\
Contemporaneous unemployment & 5.66 \\
(first month of panel) & {$[1.56]$} \\
Min unemployment during job & 4.83 \\
(until first month of panel) & {$[1.23]$} \\
\hline Data: SIPP Panels 1990 - 2001. Estimation Sample used in paper. All \\
demographics refer to first month in which the worker is observed. \\
\end{tabular}


Table A-2 : Effect of Past Labor Market Conditions on Wages

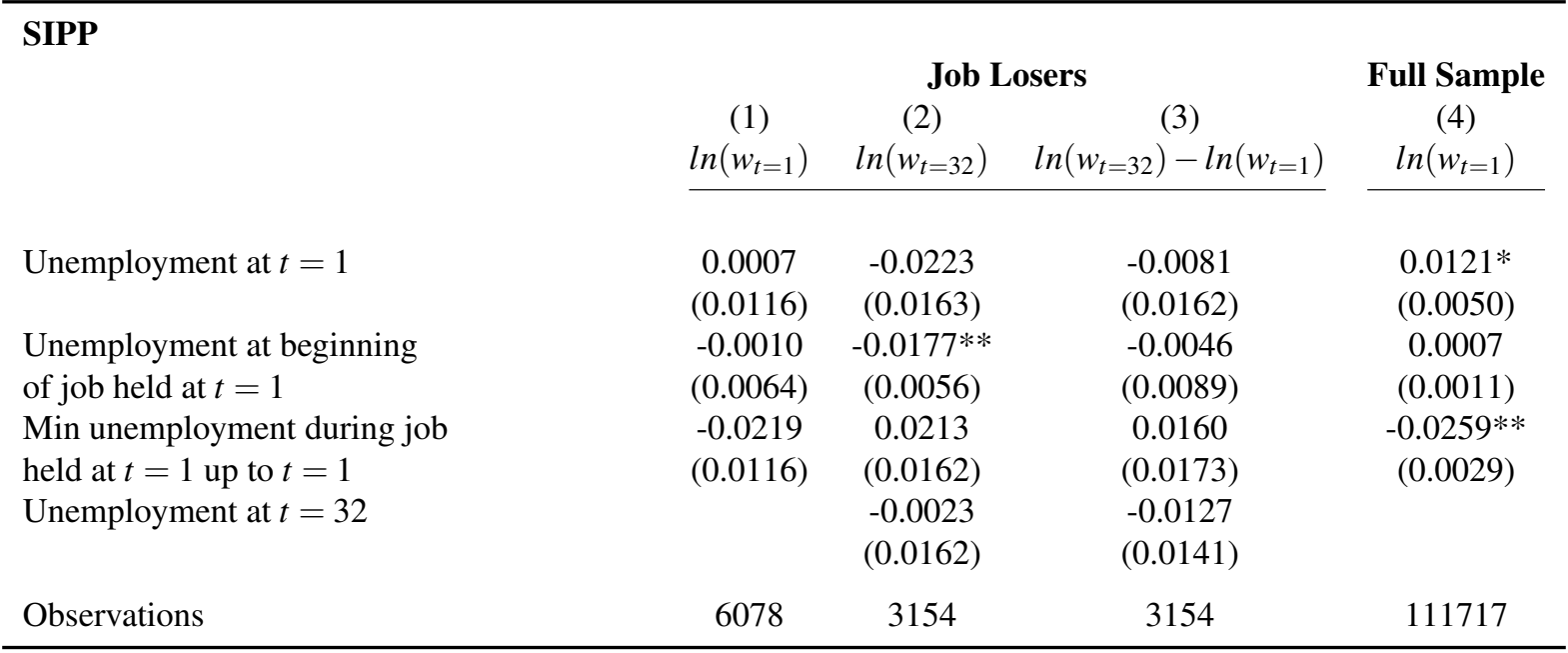

Significance Levels: $* \mathrm{p}<.05 * * \mathrm{p}<.01$, standard errors clustered on state level. Data: SIPP Panels 1990-2001. $t=1$ and $t=32$ are the 1 st and 32nd month a worker is observed in the panel. Job Losers are all workers who hold a job at $t=1$ and who are displaced within the next 32 months. The full sample consists of all workers employed at $t=1$. See Table 2 and main text for additional comments on the specification. 
Table A-3 : Effect of Past Labor Market Conditions on Wages using Level of Wages and Including Non-employed Individuals as Zeros

\begin{tabular}{|c|c|c|c|c|c|}
\hline \multicolumn{6}{|c|}{ Panel A: Dependent variable: Level of wage at lost job } \\
\hline & (1) & (2) & (3) & (4) & \\
\hline & $w_{t_{d}}$ & $w_{t_{d}}$ & $w_{t_{d}}$ & $w_{t_{d}}$ & \\
\hline Unemployment at job loss & $\begin{array}{l}-0.101 \\
(0.924)\end{array}$ & & & $\begin{array}{l}2.232 * \\
(1.103)\end{array}$ & \\
\hline $\begin{array}{l}\text { Unemployment at beginning } \\
\text { of lost job }\end{array}$ & & $\begin{array}{c}-3.051 * * \\
(0.554)\end{array}$ & & $\begin{array}{l}-0.338 \\
(1.015)\end{array}$ & \\
\hline $\begin{array}{l}\text { Min unemployment during } \\
\text { lost job }\end{array}$ & & & $\begin{array}{c}-5.852 * * \\
(1.112)\end{array}$ & $\begin{array}{c}-6.825^{* *} \\
(2.137)\end{array}$ & \\
\hline Observations & 50316 & 50316 & 50316 & 50316 & \\
\hline \multicolumn{6}{|c|}{ Panel B: Dependent variable: Level of wage at current job and change in wage levels } \\
\hline & (1) & $(2)$ & (3) & (4) & (5) \\
\hline & $w_{t}$ & $w_{t}$ & $w_{t}$ & $w_{t}$ & $w_{t}-w_{t_{d}}$ \\
\hline Unemployment at job loss & $\begin{array}{c}-2.237 * * \\
(0.813)\end{array}$ & & & $\begin{array}{c}-2.408 * * \\
(0.827)\end{array}$ & $\begin{array}{l}-2.086 \\
(1.414)\end{array}$ \\
\hline $\begin{array}{l}\text { Unemployment at beginning } \\
\text { of lost job }\end{array}$ & & $\begin{array}{c}0.232 \\
(0.609)\end{array}$ & & $\begin{array}{c}0.480 \\
(0.771)\end{array}$ & $\begin{array}{c}0.630 \\
(1.197)\end{array}$ \\
\hline $\begin{array}{l}\text { Min unemployment during } \\
\text { lost job }\end{array}$ & & & $\begin{array}{l}-0.806 \\
(1.017)\end{array}$ & $\begin{array}{l}0.0816 \\
(1.297)\end{array}$ & $\begin{array}{l}5.787^{*} \\
(2.280)\end{array}$ \\
\hline $\begin{array}{l}\text { Contemporaneous } \\
\text { unemployment }\end{array}$ & $\begin{array}{c}-4.337 * * \\
(1.241)\end{array}$ & $\begin{array}{c}-5.573 * * \\
(1.157)\end{array}$ & $\begin{array}{c}-5.540 * * \\
(1.143)\end{array}$ & $\begin{array}{l}-4.225^{* *} \\
(1.248)\end{array}$ & $\begin{array}{c}-13.61 * * \\
(1.300)\end{array}$ \\
\hline Observations & 50316 & 50316 & 50316 & 50316 & 50316 \\
\hline
\end{tabular}

Significance Levels: $* \mathrm{p}<.05 * * \mathrm{p}<.01$, standard errors clustered on state level. Specification is the same as Table 2 with the difference that the dependent variable is measured in levels rather than logs and that wages of workers who do not hold a job at time $t$, the interview time, are set to zero and included in the sample. 
Table A-4 : Effect of Past Labor Market Conditions on Wages

\begin{tabular}{|c|c|c|c|}
\hline Panel A: CPS DWS - Only v & reemploy & in same & cupation \\
\hline & (1) & (2) & (3) \\
\hline & $\ln \left(w_{t_{d}}\right)$ & $\ln \left(w_{t}\right)$ & $\ln \left(w_{t}\right)-\ln \left(w_{t_{d}}\right)$ \\
\hline Unemployment at job loss & $\begin{array}{c}0.0053 \\
(0.0058)\end{array}$ & $\begin{array}{c}-0.0163 \\
(0.0093)\end{array}$ & $\begin{array}{l}-0.0264 * \\
(0.0099)\end{array}$ \\
\hline Unemployment at beginning & -0.0066 & -0.0107 & -0.0054 \\
\hline of lost job & $(0.0050)$ & $(0.0060)$ & $(0.0054)$ \\
\hline Min unemployment during & -0.0145 & 0.0013 & 0.0171 \\
\hline lost job & $(0.0105)$ & $(0.0115)$ & $(0.0120)$ \\
\hline Contemporaneous & & -0.0047 & -0.0032 \\
\hline unemployment & & $(0.0086)$ & $(0.0096)$ \\
\hline Observations & 7851 & 9203 & 7851 \\
\hline Panel B: CPS DWS - Only v & reemploye & in same $i$ & dustry \\
\hline & (1) & (2) & (3) \\
\hline & $\ln \left(w_{t_{d}}\right)$ & $\ln \left(w_{t}\right)$ & $\ln \left(w_{t}\right)-\ln \left(w_{t_{d}}\right)$ \\
\hline Unemployment at job loss & 0.0070 & -0.0070 & -0.0129 \\
\hline & $(0.0079)$ & $(0.0107)$ & $(0.0109)$ \\
\hline Unemployment at beginning & -0.0039 & -0.0131 & -0.0116 \\
\hline of lost job & $(0.0057)$ & $(0.0083)$ & $(0.0089)$ \\
\hline Min unemployment during & $-0.0301 *$ & 0.0033 & 0.0280 \\
\hline lost job & $(0.0116)$ & $(0.0143)$ & $(0.0166)$ \\
\hline Contemporaneous & & -0.0065 & -0.0100 \\
\hline unemployment & & $(0.0102)$ & $(0.0097)$ \\
\hline Observations & 6302 & 7336 & 6302 \\
\hline
\end{tabular}

Significance Levels: $* \mathrm{p}<.05 * * \mathrm{p}<.01$, standard errors clustered on state level. Data: CPS Displaced Worker Survey 1984 - 2006. Unemployment is measured yearly on state level 
Table A-5 : Effect of Max Unemployment Rate on Wages

\begin{tabular}{lcc}
\hline Panel A: Log of wage at lost job & $(1)$ & $(2)$ \\
& $\ln \left(w_{t_{d}}\right)$ & $\ln \left(w_{t_{d}}\right)$ \\
\cline { 2 - 3 } & & \\
Unemployment at job loss & 0.0024 \\
& & $(0.0033)$ \\
Unemployment at beginning & & -0.0037 \\
of lost job & & $(0.0023)$ \\
Min unemployment during & & $-0.0252^{* *}$ \\
lost job & $(0.0044)$ \\
Max unemployment during & $-0.0088^{* * *}$ & 0.0040 \\
lost job & $(0.0029)$ & $(0.0029)$ \\
Observations & 44091 & 44091 \\
\hline Panel B: Log of wage at current job & $(1)$ & $(2)$ \\
& $\ln \left(w_{t}\right)$ & $\ln \left(w_{t}\right)$ \\
\cline { 2 - 3 } & & \\
& & $-0.0112 * *$ \\
Unemployment at job loss & & $(0.0040)$ \\
& & -0.0026 \\
Unemployment at beginning & & $(0.0037)$ \\
of lost job & & 0.0055 \\
Min unemployment during & & $(0.0062)$ \\
lost job & & -0.0037 \\
Max unemployment during & $-0.0085^{*}$ & $(0.0047)$ \\
lost job & $(0.0032)$ & 0.0041 \\
Contemporaneous & -0.0001 & $(0.0040)$ \\
unemployment & $(0.0039)$ \\
Observations & 31109 & 31109 \\
\hline
\end{tabular}

Notes: See Table 2. 
Table A-6 : Effect of Unemployment on Post-Displacement Wage by Subgroups

\section{Panel A: Log of wage at lost job}

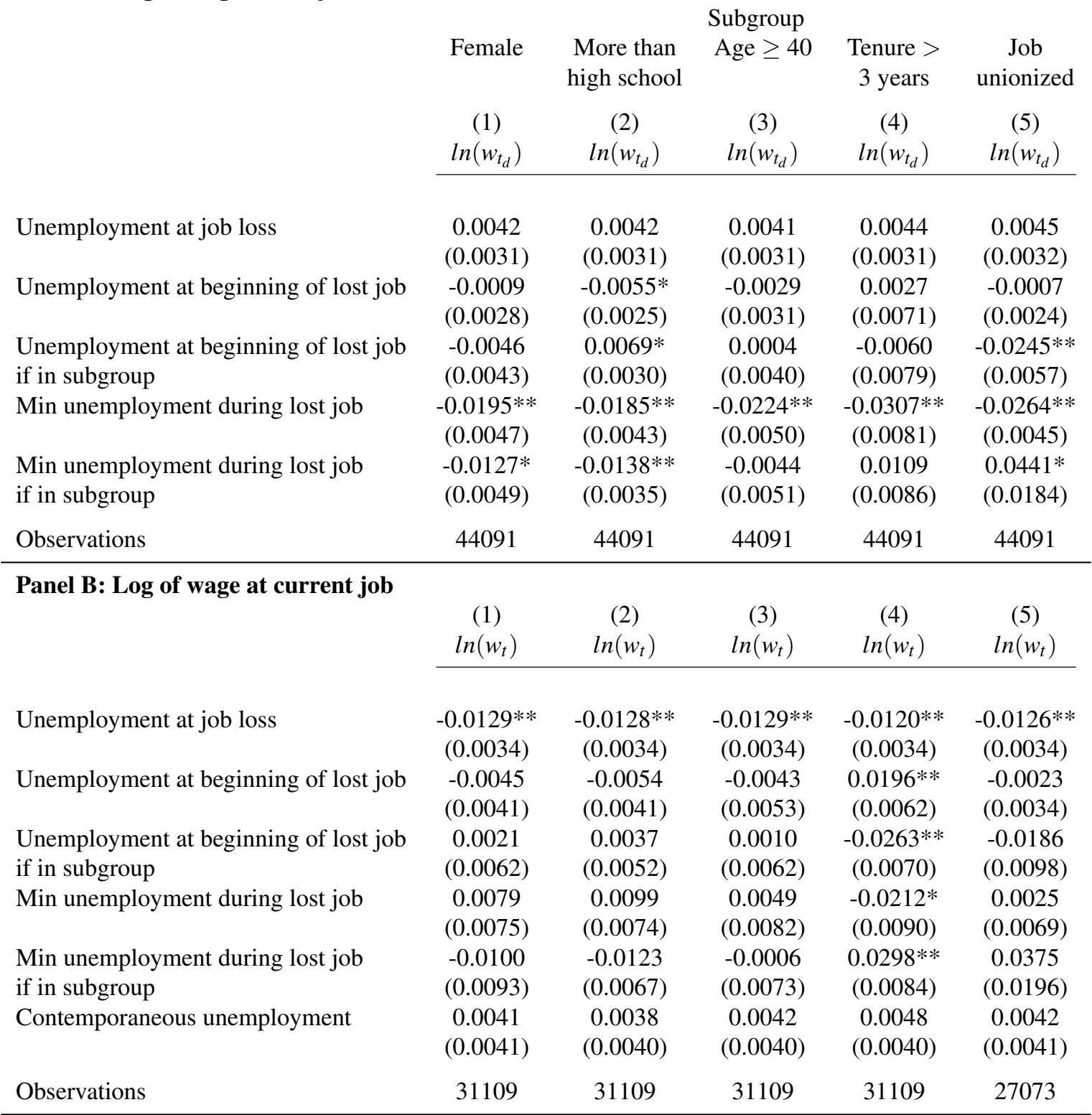

Regressions also control for current unemployment and unemployment at time of job loss. Other controls are education, experience, tenure, union at lost job, nonwhite, female, married, years since jobloss, reason for jobloss, parttime at lost job, state, year, and industry Data: CPS Displaced Worker Survey $1984-2006$, Unemployment is measured yearly on state level Significance Levels: * p $<.05 * *$ $\mathrm{p}<.01$, standard errors clustered on state level. 
Table A-7 : Effect of Unemployment - Including Interaction of Current and Minimum Unemployment Rate

Dep. variable: Log of wages at current job

(1)

(2)

(3)

(4)

$\ln \left(w_{t}\right)$
$-0.0134^{* *}$
$(0.0030)$

$\ln \left(w_{t}\right)$

$\ln \left(w_{t}\right)$

$\ln \left(w_{t}\right)$

Unemployment at job loss

$(0.0030)$

Unemployment at beginning

of lost job

Min unemployment during

lost job

Contemporaneous unemployment

$\begin{array}{cccc} & (0.0027) & & \\ & & -0.0355^{* *} & -0.0031) \\ 0.0015 & -0.0033 & -0.0104) & (0.0125) \\ (0.0044) & (0.0055) & (0.0104) & -0.0185 \\ 0.0006 & 0.0002 & 0.0039^{* *} & (0.0106) \\ (0.0005) & (0.0007) & (0.0012) & 0.0038^{* *} \\ 3110 & & 0.0012)\end{array}$

$-0.0040$

(0.0034)

$-0.0038$

$(0.0027)$

$(0.0031)$

Min. unemployment $x$

31119

31109

31119

31109

\section{Dep. variable: Change in log wage}

$$
\text { (1) }
$$

(3)

$$
\ln \left(w_{t}\right)-\ln \left(w_{t_{d}}\right) \quad \ln \left(w_{t}\right)-\ln \left(w_{t_{d}}\right) \quad \ln \left(w_{t}\right)-\ln \left(w_{t_{d}}\right) \quad \ln \left(w_{t}\right)-\ln \left(w_{t_{d}}\right)
$$

Unemployment at job loss

Unemployment at beginning

of lost job

Min unemployment during

lost job

Contemporaneous unemployment

Min. unemployment $\times$

contemporaneous unemployment

Observations

$\begin{array}{cccc}-0.0064 & & & -0.0094^{*} \\ (0.0038) & & & (0.0043) \\ & 0.0032 & & -0.0011 \\ & (0.0029) & 0.0225^{*} & (0.0033) \\ & & (0.0109) & 0.0302^{*} \\ -0.0385^{* *} & -0.0361^{* *} & -0.0230^{*} & -0.0119) \\ (0.0039) & (0.0043) & (0.0087) & (0.0087) \\ 0.0021^{* *} & 0.0012^{*} & -0.0011 & -0.0012 \\ (0.0005) & (0.0005) & (0.0013) & (0.0013) \\ 27081 & 27073 & 27081 & 27073\end{array}$

Regressions also control for education, experience, tenure, union at lost job, nonwhite, female, married, years since jobloss, reason for jobloss, parttime at lost job, state, year, and industry Data: CPS Displaced Worker Survey 1984 - 2006, Unemployment is measured yearly on state level Significance Levels: * $\mathrm{p}<.05 * * \mathrm{p}<.01$, standard errors clustered on state level. 
Table A-8 : Effect of Past Labor Market Conditions on Wages - Proxying Labor Market Tightness with Log-Deviations from Industry Employment Trend

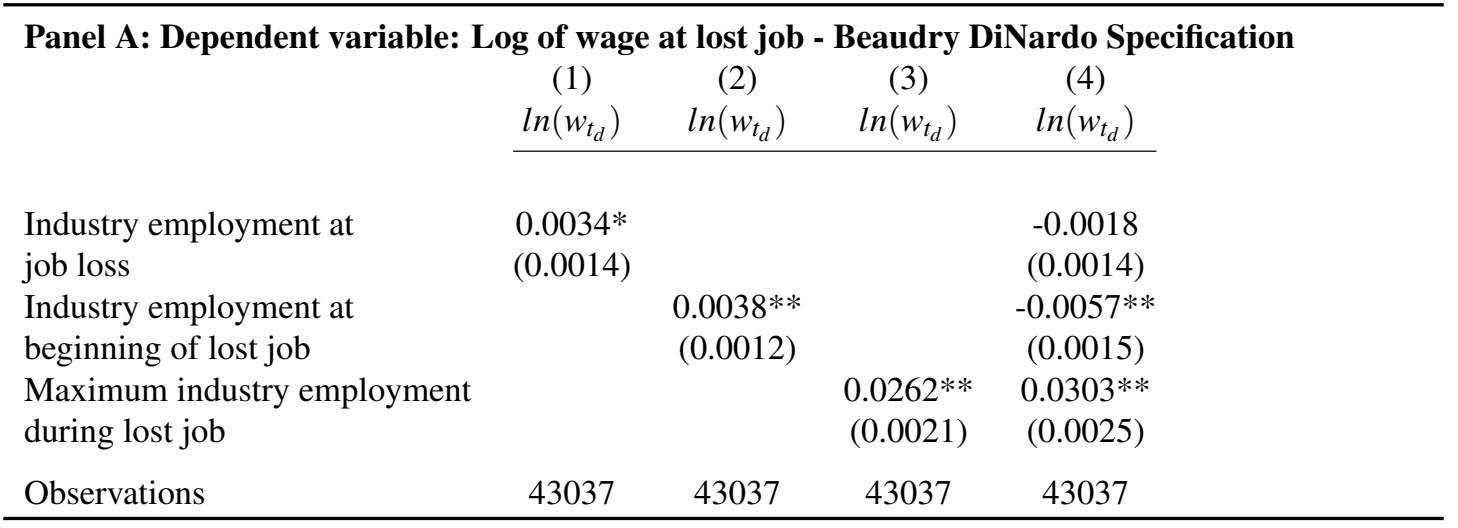

Panel B: Dependent variable: Log of wage at current job and change in log wage

$\begin{array}{llll}(1) & \text { (2) (3) } \quad \text { (4) }\end{array}$

$\begin{array}{llll}\ln \left(w_{t}\right) \quad \ln \left(w_{t}\right) & \ln \left(w_{t}\right) \quad \ln \left(w_{t}\right) \quad \underline{\ln \left(w_{t}\right)-\ln \left(w_{t_{d}}\right)}\end{array}$

Industry employment at

$-0.0007$

$(0.0020)$

Industry employment at

beginning of lost job

Maximum industry employment

during lost job

Contemporaneous

industry employment

Observations

$\begin{array}{ccccc}(0.0020) & & & (0.0021) & (0.0019) \\ & -0.0006 & & -0.0022 & 0.0024 \\ & (0.0015) & & (0.0020) & (0.0022) \\ & & 0.0029 & 0.0048 & -0.0252 * * \\ -0.0023 & -0.0024 & -0.0021 & -0.0021 & (0.0039) \\ (0.0042) & (0.0042) & (0.0043) & (0.0042) & (0.0043) \\ 29966 & 29966 & 29966 & 29966 & 26128\end{array}$

Significance Levels: $* \mathrm{p}<.05 * * \mathrm{p}<.01$, standard errors clustered on state level. The specifications are the same as in Table 2 except (log) deviations from industry employment trend rather than the unemployment rate is used as the measure for labor market tightness. Industry employment is on the level of the 9 BLS super sectors. Industry employment is measured as log deviations from trend employment (i.e. the Hodrick-Prescott smoothed employment series) multiplied with 100, so the coefficients are on the same scale as Table 2 . 
Table A-9 : Selection - The Correlation of Observables with Labor Market History

\begin{tabular}{lcccccc}
\hline & $\begin{array}{c}(1) \\
\text { Education }\end{array}$ & $\begin{array}{c}(2) \\
\text { Experience }\end{array}$ & $\begin{array}{c}(3) \\
\text { Married }\end{array}$ & $\begin{array}{c}(4) \\
\text { Nonwhite }\end{array}$ & $\begin{array}{c}(5) \\
\text { Female }\end{array}$ & $\begin{array}{c}(6) \\
\text { Lost/Left Job } \\
\text { Unionized }\end{array}$ \\
\cline { 2 - 7 } & & & & & & \\
Unemp at job loss & 0.0067 & 0.0591 & -0.0003 & 0.0009 & -0.0031 & -0.0025 \\
Unemp at beginning of lost job & $(0.0115)$ & $(0.0473)$ & $(0.0018)$ & $(0.0016)$ & $(0.0017)$ & $(0.0015)$ \\
& 0.0098 & 0.0472 & -0.0006 & -0.0011 & 0.0025 & 0.0020 \\
Min unemp during lost job & $(0.0085)$ & $(0.0451)$ & $(0.0016)$ & $(0.0013)$ & $(0.0020)$ & $(0.0012)$ \\
Contemporaneous Unemployment & -0.0207 & -0.1416 & -0.0003 & -0.0029 & -0.0014 & -0.0002 \\
& $(0.0192)$ & $(0.0885)$ & $(0.0028)$ & $(0.0022)$ & $(0.0026)$ & $(0.0024)$ \\
Observations & $-0.0416^{* *}$ & $-0.1249^{*}$ & 0.0012 & 0.0003 & -0.0035 & -0.0001 \\
& $(0.0126)$ & $(0.0603)$ & $(0.0024)$ & $(0.0017)$ & $(0.0024)$ & $(0.0018)$ \\
\hline
\end{tabular}

Significance Levels: $* \mathrm{p}<.05 * * \mathrm{p}<.01$, standard errors clustered on state level. The specifications are the same as in Table 2 except the dependent variables are changed. The dependent variable in each column is dropped from the controls. 